\title{
The application of $x$-ray diffraction and electron microscopy to the study of human peripheral nerve obtained by biopsy
}

\author{
J. B. FINEAN AND A. L. WOOLF \\ From the Department of Medical Biochemistry and Pharmacology, University of \\ Birmingham, and the Midland Centre for Neurosurgery, Smethwick
}

SYNOPSIS Human peripheral nerves obtained by biopsy from patients suffering from neuro: muscular disorders have been studied by $x$-ray diffraction and electron microscopy. Few abnorma $\$$ diffraction patterns have yet been recorded and their significance is not yet established. Electron micrographs have revealed wide variations in the numbers of myelinated fibres included in the nerve trunks and have facilitated a detailed study of the Schwann cell-axon relationships in the large numbers of unmyelinated fibres always present. Some indications of demyelination have. been encountered.

In the past, the range of abnormalities detectable in biopsy specimens of cutaneous nerves has been severely limited. Morphological changes detectable by the light microscope have been largely restricted to degenerative swelling of myelin sheaths and axons, loss of myelin as observed in 'myelin preparations', and loss of non-myelinated fibres as seen in silver preparations. Little information relating to the Schwann cells could be obtained and, as will be shown, the smallest axons could not be seen with the light microscope. Furthermore, little structural detail could be discerned in individual nerve fibres. This applies particularly to axons, which stain almost uniformly in silver preparations.

This paper reports a preliminary attempt to extend the scope of biopsy studies of peripheral nerve by introducing $x$-ray diffraction and electron microscope techniques.

\section{MATERIALS AND METHODS}

The specimen studied was almost invariably a small branch of the medial antebrachial cutaneous nerve of the forearm taken in the course of muscle biopsy in a variety of neuromuscular diseases. In only a few cases was there any evidence of disturbed cutaneous sensation.

A $2 \mathrm{~cm}$. length of nerve was isolated with great care and cut into four pieces which were immediately immersed in chilled saline or fixative. The specimen for diffraction studies was immersed in $0.9 \%$ saline, those for electron microscopy in $2 \% \mathrm{OsO}_{4}$ in veronal-acetate Received for publication 18 September 1960. buffer, $p \mathrm{H} 7$, or $1 \% \mathrm{KMnO}_{4}$ in veronal-acetate buffer $\frac{2}{2}$ $p \mathrm{H} \mathrm{7,} \mathrm{that} \mathrm{for} \mathrm{paraffin} \mathrm{embedding} \mathrm{for} \mathrm{light} \mathrm{microscop}$ in the buffered osmic acid, and that for haematoxyli and eosin staining for light microscopy in $10 \%$ formo saline.

So far, specimens of nerve from 75 patients have bee? made available for $x$-ray diffraction and electron micro scope studies.

The nerve selected for diffraction studies was stored a about $4^{\circ} \mathrm{C}$. until the diffraction pattern could be recordedo The delay in obtaining the diffraction pattern was neve? more than six hours and frequently less than one hour As a check on the possibility of degenerative changes taking place during the period of delay before recording the diffraction patterns, rat sciatic nerves were kept under similar conditions for comparable periods, and the diffraction patterns subsequently obtained were com $\rightarrow$ pared with those from freshly isolated preparations. I addition, specimens of human nerve which had been examined very soon after isolation were frequently store $\$$ and re-examined after several hours to see if such storage resulted in a change in diffraction pattern.

For the purpose of recording the diffraction patterns the nerve samples were enclosed in close-fitting, thine walled, glass capillary tubes filled with physiologics saline. A low-angle $x$-ray diffraction camera of a typ $\overline{8}$ previously described (Finean, 1956) was used, and $a^{+}$ standard exposure of one hour at a specimen to filmp distance of about $150 \mathrm{~mm}$., using a slit collimatin system, was adopted.

Specimens for electron microscopy were fixed for tw hours in $\mathrm{OsO}_{4}$ or three hours in $\mathrm{KMnO}_{4}$, washed iff saline, and left in $70 \%$ ethyl alcohol at about $4{ }^{\circ} \mathrm{C}$. over night. The dehydration was completed in $90 \%$ ang 


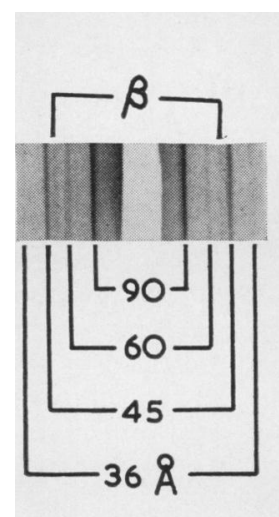

a

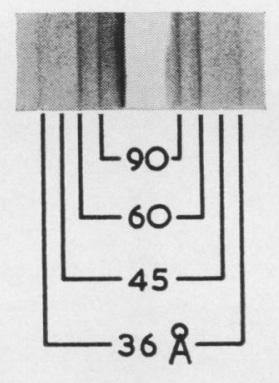

b

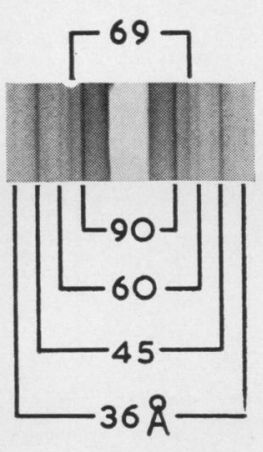

c

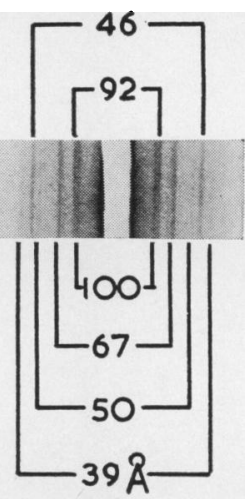

d

Electron micrographs were selected from studies relating to five Werdig cases, namely, Case 1, dermatomyositis, Case 2, Werdig-Hoffmann disease, Case 3, an obscure, slowly progressive peripheral neuropathy, Case 4, sensory neuropathy, and Case 5, dystrophia myotonica.

FIG. 1. Low angle $x$-ray diffraction patterns obtained from human peripheral nerve. $a$, Normal pattern; $b, c, d, a b n o r m a l$ patterns. The numbers refer to the diffraction spacings calculated for each band in the pattern assuming that they arise from the CuKa radiation $(\lambda=1.54 A)$. They are grouped so as to distinguish bands related to the main fundamental spacings, i.e., those below the pattern. As unfiltered $C u$ radiation was used in these experiments diffraction bands related to $C u K \beta$ radiations $(\lambda=1 \cdot 39 \AA)$ can occasionally be distinguished. Such a diffraction is indicated as $\beta$ in a, and corresponds to the $45 \AA$ diffraction.

absolute alcohols, and the specimens were embedded in Araldite casting resin. Thin sections were cut with the aid of Porter-Blum or Philips ultramicrotomes and examined in a Siemens electron microscope, type Elmiskop I.

\section{RESULTS}

Little reference will be made to the conditions from which the patients were suffering, as in this preliminary report the object is simply to show the scope of the methods and their potential value rather than to attempt to identify pathological changes. A more detailed treatment of individual cases will be included in case reports which will be published separately.

X-RAY DIFFRACTION Of the 75 nerve specimens examined by low-angle diffraction methods, 45 gave a pattern which, from a comparison with those of other mammalian peripheral nerve (Finean, 1960) such as rat sciatic nerve, could be regarded as normal. This pattern showed four reflections which could be accounted for as the second, third, fourth, and fifth order diffractions of a fundamental period of $180 \AA$. The first order diffraction was not readily detectable under the conditions used in these experiments. An example of such a diffraction pattern is reproduced in Fig. 1a.

Of the remaining nerve specimens, 17 yielded no detectable diffraction and 13 gave abnormal patterns.
The most common abnormality was an increased intensity of the third order diffraction accompanied by a decrease of the fourth order (Fig. 1b), but a few preparations gave diffraction spacings (Fig. 1c and 1d) not present in the normal pattern. No straightforward relationship between these abnormal patterns and the clinical condition of the patients from whom the nerves were taken is yet apparent.

ELECTRON MICROSCOPY So far about 50 of the preparations have yielded useful electron microscope data but only 10 have been studied in detail.

All preparations which had given intense diffraction patterns yielded electron micrographs showing large numbers of well-myelinated nerve fibres, always accompanied by a moderate proportion of unmyelinated ones.

In general, there was a close correspondence between the intensity of the diffraction pattern of the fresh tissue and the abundance of myelinated fibres as seen in electron micrographs of nerve crosssections, although in a few cases myelinated fibres were detected in electron microscope preparations when the equivalent $x$-ray diffraction specimen had yielded no pattern.

The myelin layers, as seen in both $\mathrm{OsO}_{4}$-fixed and in $\mathrm{KMnO}_{4}$-fixed preparations, showed exactly the same features as have already been described for other mammalian peripheral nerves (Fernández- 

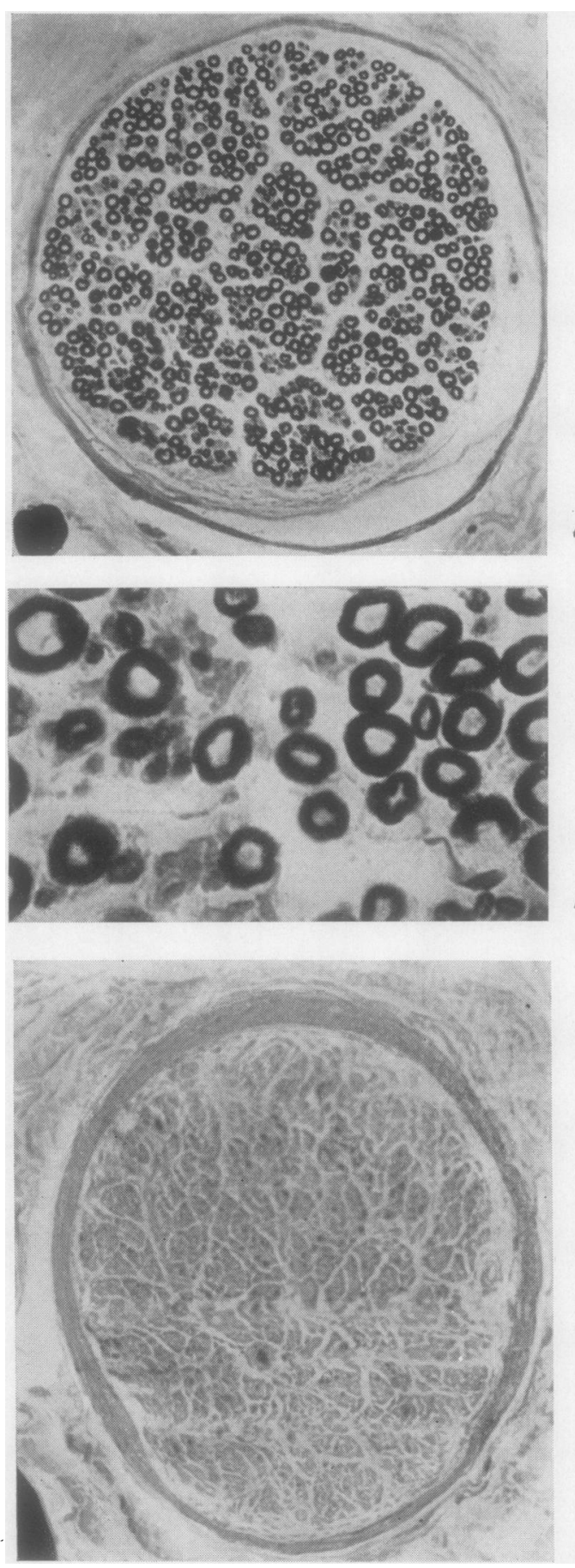

FIG. 2. Photomicrographs obtained from cross sections of $\mathrm{OsO}_{4}$-fixed and paraffin-embedded preparations of biopsy material. a Case $1 \times 200, b$ Case $1 \times 900, c$ Case $4 \times 400$
Morán, 1957; Fernández-Morán and Finean, 1957; Finean and Robertson, 1958; Robertson, 1959, 1960; Sjöstrand, 1960). The continuity between the Schwann cell membrane and the myelin layers (the mesaxon) was frequently observed, and it was clear that the process of myelination had been essentially the same as has been established for other species (Geren, 1954; Robertson, 1959; Peters and Muir, 1959). The relationships between nerve axons and Schwann cells in the unmyelinated fibres were also similar to those previously reported (Causey, 1960; Gasser, 1958; Robertson, 1959).

The variation in the proportion of myelinated to unmyelinated fibres provided the most striking feature of these electron microscope studies. In several preparations very few myelinated fibres were to be seen, and in one case none at all. Unmyelinated fibres, however, still appeared numerous, and there was an apparently normal relationship between axons and Schwann cells. Many of these poorly myelinated nerve specimens will be considered in greater detail in other publications but some electron micrographs relating to a few cases are reproduced here in order to illustrate the general points made.

Fig. 3 shows low-magnification electron micrographs selected to illustrate the variations in the composition of nerve bundles encountered in these studies. Fig. 3a is taken from an adult nerve containing numerous well-myelinated fibres. Photomicrographs from the same tissue are shown in Fig. $2 a$ and $b$.

In this preparation the myelinated nerve fibres accounted for at least $50 \%$ of the tissue volume but unmyelinated fibres were freely interspersed, frequently concentrated into large bundles.

In specimens taken from infants under 1 year old myelinated fibres were far less abundant (Fig. 3b), and both axons and myelin sheaths were much smaller than in the adult tissue. Unmyelinated fibres appeared to be far more numerous but the effect was certainly enhanced by the fact that myelinated fibres were small and accounted for only a small proportion of the tissue volume.

Fig. 3c shows only a few myelinated fibres but this micrograph was obtained from a specimen taken from an adult suffering from an obscure, slowly progressive, peripheral neuropathy. A striking feature of the micrographs obtained from this preparation is the prominence of numerous large axons surrounded by regions of unusually low density. The distribution of such regions is reminiscent of the distribution of well-myelinated fibres in normal adult tissue, and these low density regions may well reflect the degeneration of large fibres (see also Bunge, Bunge, and Ris, 1960). Fig. 6b is a 

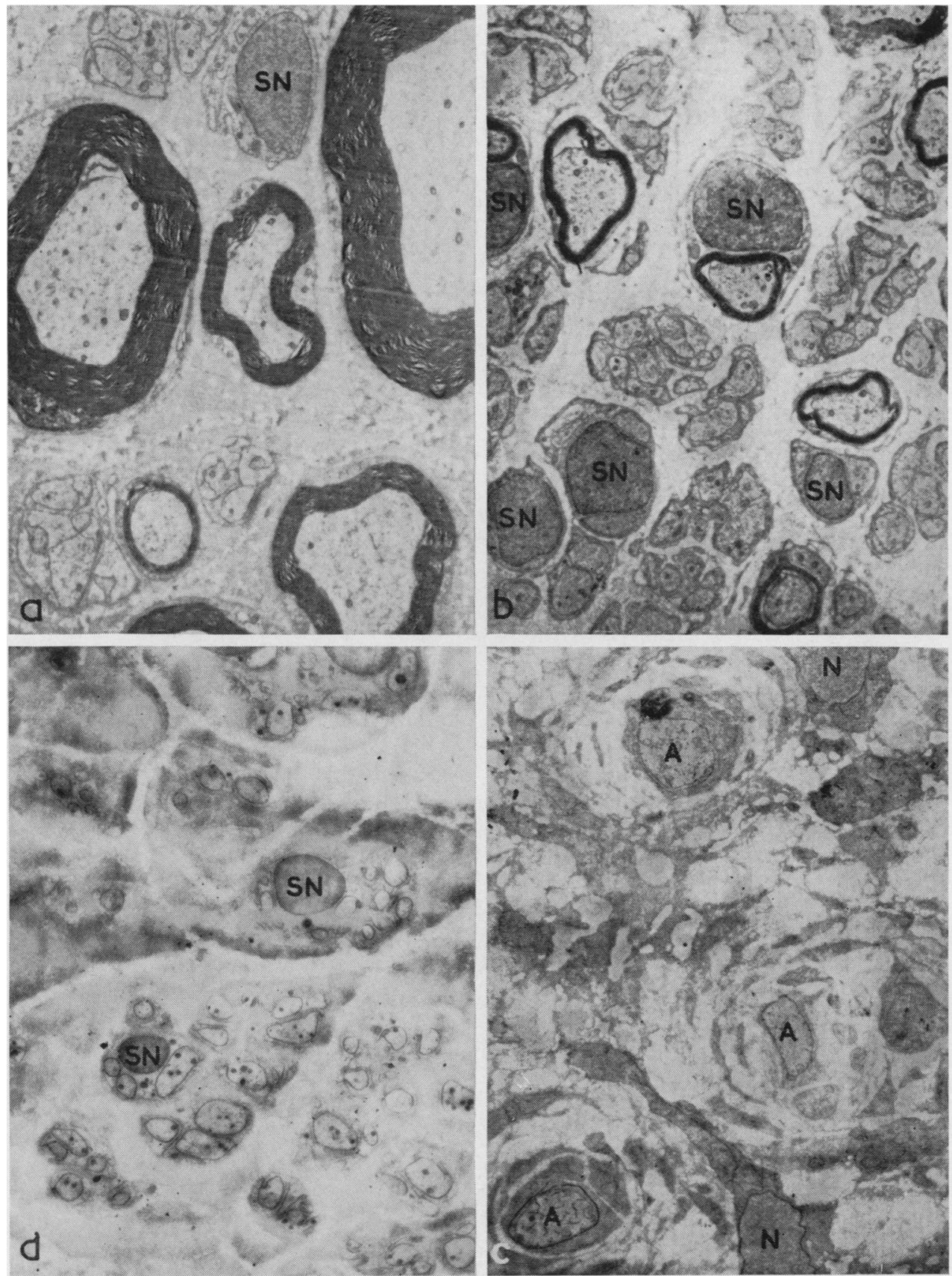

FIG. 3. Low magnification $(\times 5,000)$ electron micrographs of cross sections obtained from biopsy specimens. A number of Schwann cell nuclei $(S N)$, unidentified nuclei $(N)$, and axons $(A)$ are labelled. $a$ Case $1 \mathrm{OsO}_{4}$-fixed $b$ Case $2 \mathrm{OsO}_{4}$-fixed $c$ Case $3 \mathrm{KMnO}_{4}$-fixed $d \mathrm{Case}_{4} \mathrm{OsO}_{4}$-fixed 

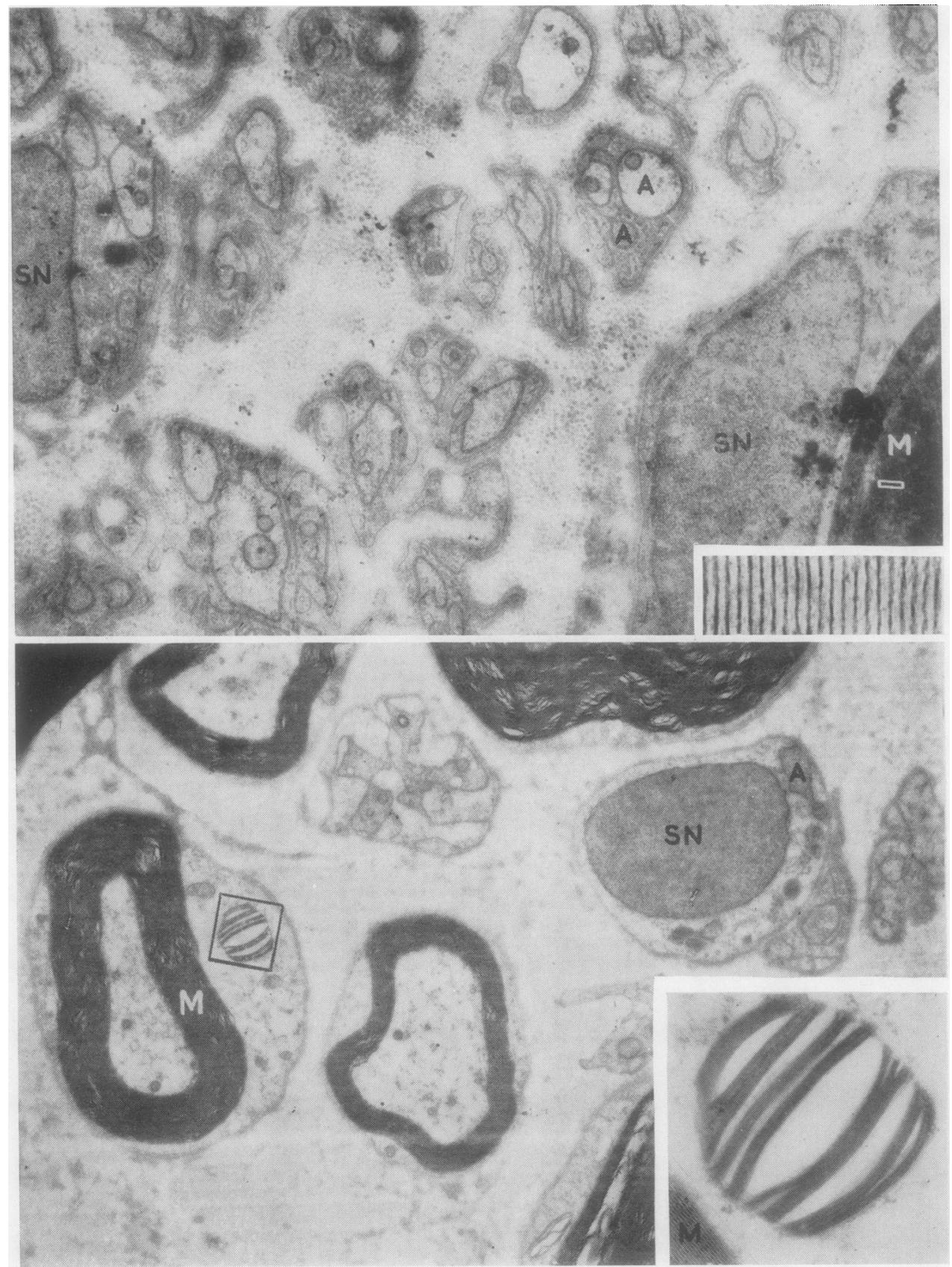

FIG. 4. Electron micrograph $(\times 15,000)$ from Case 5 showing a portion of a large myelinated fibre $(M)$ (inset myelin layers $\times 160,000)$ and a group of unmyelinated fibres with examples of large and small axons $(A)$.

FIG. 5. Electron micrograph $(\times 10,000)$ from Case 1 showing a typical 'normal' distribution of myelinated and unmyelinated fibres and including an unusual layered body (inset $\times 40,000$ ). 

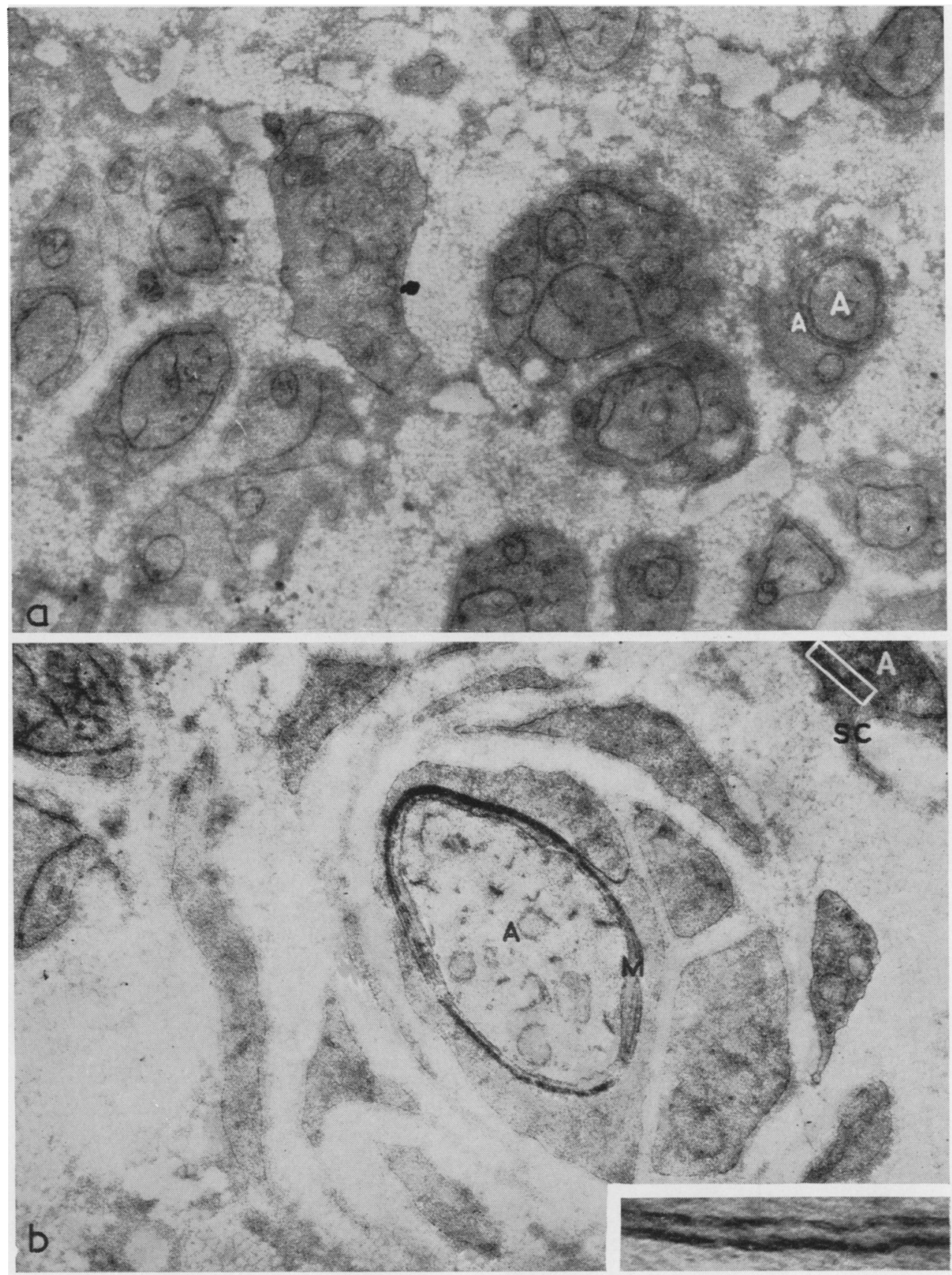

FIG. 6. Electron micrographs from Case 3. a A group of unmyelinated fibres $\times 15,000$. b A sparsely myelinated fibre located in a region of low density. $\times 40,000$. Inset is a high magnification $(\times 160,000)$ electron micrograph showing detailed structure of apposed axon and Schwann cell membranes. 

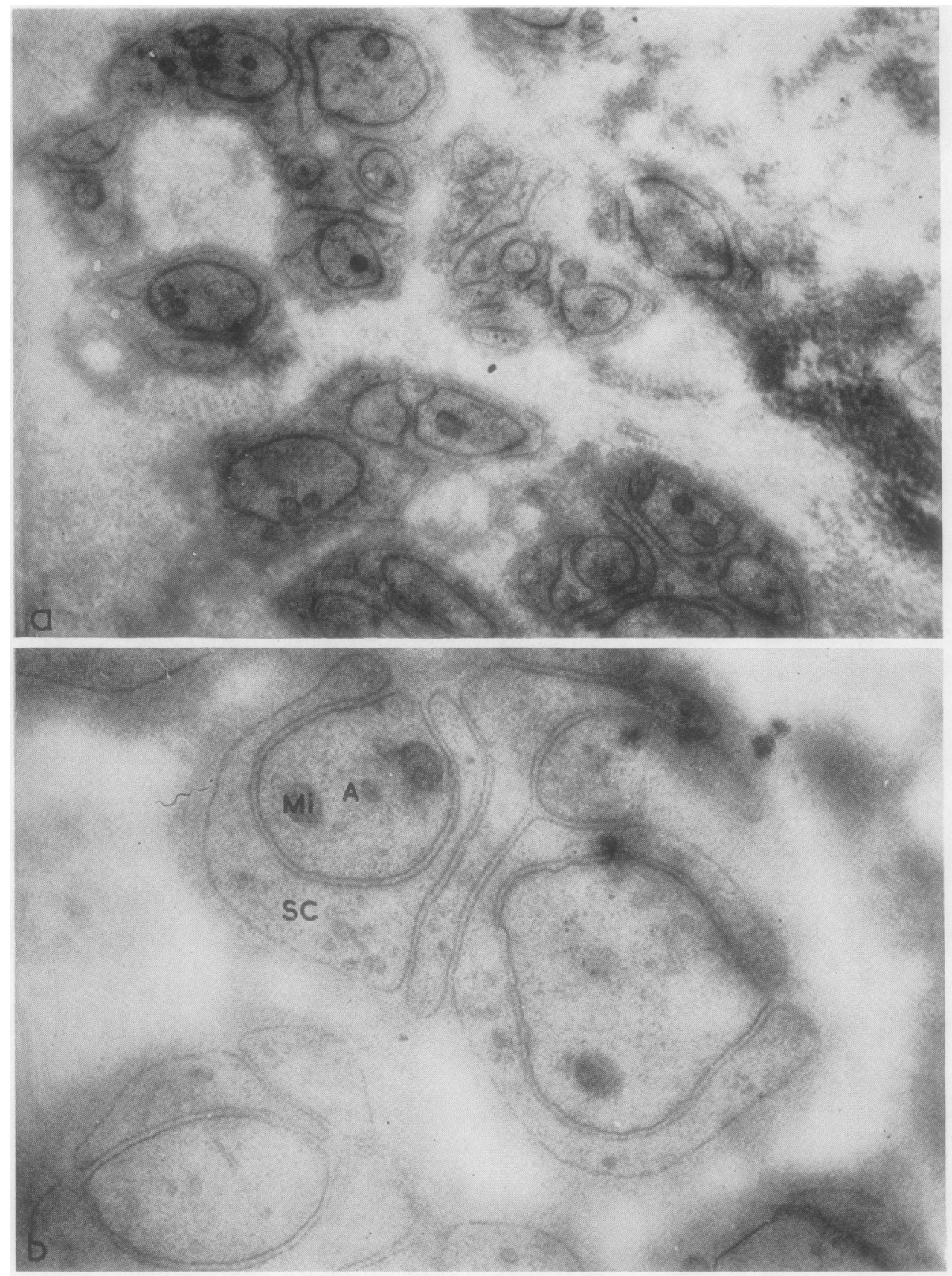

FIG. 7. Electron micrographs from Case 4. a Unmyelinated fibres $\times 20,000 . \quad b$ Unmyelinated fibres $\times 60,000$. In one nerve fibre Schwann cell (S.C.), axon (A), and mitochondria (Mi) are distinguished. 
relatively high-magnification electron micrograph which shows one of the few myelinated fibres detected in this case. Very few myelin layers surround the axon, and the general appearance is that of a nerve in an early stage of myelination. However, the region around the nerve fibre shows an unusual array of profiles, presumably of cell processes, and also a low density such as has already been considered as possibly arising from a demyelination of some of the larger axons. This fibre may, in fact, be in the process of remyelination. Unmyelinated fibres are also present, and their distribution is similar to that of the normal adult tissue. A group of such fibres is shown in Fig. 6a.

A case of sensory neuropathy in which light microscopy, electron microscopy, and $x$-ray diffraction all showed a complete absence of myelinated fibres is illustrated in Figs. 2c, 3d, and 7. Unmyelinated fibres are numerous, but they are more widely distributed than in the case illustrated in Fig. 3b. There are no obvious signs of degenerative changes, but again this is an adult nerve and the clinical history suggests that an extremely slow degeneration has probably taken place. The higher magnification electron micrographs (Fig. 7) show a variety of apparently normal axon-Schwann cell relationships among the unmyelinated fibres and include some examples of a single axon embedded in a Schwann cell, a situation that might be expected to lead to myelination in normal circumstances.

The higher magnification electron micrographs also serve to illustrate some of the more detailed structural features revealed in biopsy preparations by the application of electron microscopy.

Detail of the myelin layering is readily observed (Fig. 4 inset) but so far no abnormalities of layering have been detected in these biopsy specimens. Electron microscope preparations corresponding to those fresh nerve specimens which had yielded abnormal diffraction patterns showed no comparable abnormalities in the appearance of the myelin layers. However, one unusual feature which has been observed in several of the human specimens is a layered body, usually of circular or elliptical outline, which is located in the Schwann cell cytoplasm adjacent to the myelin sheath (Fig. 5). The significance of this body is not yet understood.

Electron microscopy has also revealed the presence of minute axons included in the bundles of unmyelinated fibres (Figs. 4, 6a, and 7a). It is possible to examine in detail the relationships between the surfaces of the axons and the Schwann cell, and the inset in Fig. 6b shows the detail of closely apposed axon and Schwann cell surfaces as revealed in $\mathrm{KMnO}_{4}$-fixed preparations. The interaction of Schwann cell and axon is the central theme of the myelination process and is the main object of attention in the present studies of abnormal nerve specimens.

\section{DISCUSSION}

X-RAY DIFFRACTION The low-angle $x$-ray diffraction technique would appear to provide a rapid method of assessing the extent of myelination in a nerve trunk. In the present studies, the exposure given was one hour and the starting of the exposure was often delayed for technical reasons but it is possible to obtain an adequate diffraction pattern in about 10 minutes, and there is no reason why this exposure should not be made immediately after the nerve has been isolated.

The significance of the abnormal diffraction patterns is still uncertain. An intensification of the third order diffraction such as some of the human preparations revealed can be produced experimentally by changing the tonicity of the immersion medium and even by leaving nerve preparations in physiological $(0.9 \%)$ saline for long periods. However, it should not occur in normal preparations within six hours if stored under favourable conditions. The results so far would therefore suggest that in some cases the myelin is either already modified before isolation or is in a condition such that deterioration occurs rapidly after isolation, even if stored under favourable conditions. The absence of corresponding abnormalities in the myelin layers as seen in the electron micrographs might mean that the changes have taken place after isolation. However, no definite conclusions will be drawn until further examples have been rigorously investigated. A similar situation also exists in relation to the few preparations which have yielded diffraction spacings not present in the 'normal' pattern.

The first conclusion to be drawn from the electron microscope studies is that the structure and method of formation of the myelin sheath in human peripheral nerve is essentially similar to that already well established for other species. The axon-Schwann cell relationships observed in the case of the unmyelinated fibres is also of the type previously described for peripheral nerve from many species.

From the point of view of the study of human nerve fibres, both for the functional correlations and for the recognition of pathological changes, the most striking features are the complex pattern of distribution of myelinated and unmyelinated fibres and the amazing variety of detail presented by the Schwann cell-axon interactions among the unmyelinated fibres. The myelinated fibres in normal adult tissue show a fairly sharp distinction between large, well-myelinated fibres and groups of smaller 
fibres with relatively thin myelin sheaths. Among the unmyelinated fibres there is a remarkable variation in the size of the axons and the numbers enfolded by each Schwann cell. These morphological variations may be related to different functions, but we do not yet know how to recognize by these structural features or by any other means the fibres transmitting pain, thermal and touch sensations, or those forming part of the autonomic nervous system. Such correlations can hardly be obtained through studies in animals, where the assessment of sensory function is almost impossible and the delicate interruption by disease of individual modalities of sensation virtually unrecognizable. Such information may eventually be obtained through correlations of electron microscope data obtained from human biopsy material with analyses of the reactions of the patients to various stimuli, but a great deal of systematic work will be required before any significant conclusions can be reached. It is hoped that our findings will be considered encouraging evidence of the potential value of electron microscopy in the pursuit of such correlations.

Our thanks are due to the following who have allowedo us to show photographs of specimens taken frome their patients: Drs. E. R. Bickerstaff, Gilbert Hall J. MacDonald Holmes, Michael Jefferson, and J. Malpas $\overrightarrow{\mathbb{\phi}}$

\section{REFERENCES}

Bunge, R. P., Bunge, M. B., and Ris, H. (1960). J. biophys. biochem $\overrightarrow{0}$ Cytol., 7, 685 .

Causey, G. (1960). The Cell of Schwann. Livingstone, Edinburgh. Fernández-Morán, H. (1957). In Metabolism of the Nervous System Wu pp. 1-34, ed. D. Richter. Pergamon Press, London.

, and Finean, J. B. (1957). J. biophys. biochem. Cytol., 3, 725.

Finean, J. B. (1956). J. sci. Instrum., 33, 161.

- (1960). In Modern Scientific Aspects of Neurology, p. 232, ed $\vec{A}$ J. N. Cumings. Arnold, London.

- , and Robertson, J. D. (1958). Brit. med. Bull., 14, 267.

Gasser, H. S. (1958). Exp. Cell Res., Suppl. 5, 3.

Geren, P. B. (1954). Ibid, 7, 558.

Robertson, J. D. (1959). Biochem. Soc. Symposia, No. 16, p. 3. (1960). In Molecular Biology, p. 87, ed. D. NachmansohnAcademic Press, New York.

Sjöstrand, F. S. (1960). In Modern Scientific Aspects of Neurology p. 188, ed. J. N. Cumings. Arnold, London. 